\title{
Machine Learning for Additive Manufacturing
}

\author{
Dean Grierson*(D), Allan E. W. Rennie and Stephen D. Quayle (D)
}

Engineering Department, Lancaster University, Lancaster LA1 4YW, UK; a.rennie@lancaster.ac.uk (A.E.W.R.); s.quayle@lancaster.ac.uk (S.D.Q.)

* Correspondence: d.grierson@lancaster.ac.uk

Definition: Additive manufacturing (AM) is the name given to a family of manufacturing processes where materials are joined to make parts from 3D modelling data, generally in a layer-upon-layer manner. AM is rapidly increasing in industrial adoption for the manufacture of end-use parts, which is therefore pushing for the maturation of design, process, and production techniques. Machine learning (ML) is a branch of artificial intelligence concerned with training programs to self-improve and has applications in a wide range of areas, such as computer vision, prediction, and information retrieval. Many of the problems facing AM can be categorised into one or more of these application areas. Studies have shown ML techniques to be effective in improving AM design, process, and production but there are limited industrial case studies to support further development of these techniques.

Keywords: machine learning; supervised learning; unsupervised learning; reinforcement learning; additive manufacturing; design for additive manufacturing; additive manufacturing process; additive manufacturing monitoring

Citation: Grierson, D.; Rennie,

A.E.W.; Quayle, S.D. Machine

Learning for Additive Manufacturing. Encyclopedia 2021, 1, 576-588. https:// doi.org/10.3390/encyclopedia1030048

Academic Editors: Raffaele Barretta, Ramesh Agarwal, Krzysztof Kamil Żur and Giuseppe Ruta

Received: 16 June 2021

Accepted: 14 July 2021

Published: 19 July 2021

Publisher's Note: MDPI stays neutral with regard to jurisdictional claims in published maps and institutional affiliations.

Copyright: (C) 2021 by the authors. Licensee MDPI, Basel, Switzerland. This article is an open access article distributed under the terms and conditions of the Creative Commons Attribution (CC BY) license (https:// creativecommons.org/licenses/by/ $4.0 /)$.

\section{Introduction}

Additive manufacturing (AM) is the name given to a family of manufacturing processes where materials are directly joined to make parts from 3D modelling data [1]. This is generally done in discrete planar layers, but non-planar processes also exist [2]. AM enables various advantages, particularly when compared with traditional manufacturing techniques, the enablement of mass part customisation and greater part complexity on the macro-, meso-, and micro-scales [3]. Other advantages include not requiring any hard tooling and enablement of on-demand manufacturing [4]. Despite these benefits, drawbacks include a lack of inherent repeatability [5] which has led to difficulty in gaining certification in some sectors [3]. Another drawback is the lack of widespread design knowledge and tools tailored specifically for AM and the above-mentioned benefits that are enabled [3].

While, to some extent, applicable to all major industries, AM development has been largely driven by the aerospace, automotive and medical sectors [4]. The major driver in the aerospace and automotive sectors is to reduce component mass whilst not hindering performance [4]. A wider range of motivations is seen for medical applications of AM, although patient customisation, improved biocompatibility and performance is a common theme [4]. AM is also often used in consumer products, with mass customisation and light-weighting both being common motivations [4]. Machine learning (ML) is a branch of artificial intelligence concerned with training programs to automatically improve their performance. With this broad definition in mind, there are different types of ML which may be classified as supervised, unsupervised, semi-supervised, or reinforcement learning [6]. Shinde and Shah identified five key application domains for ML [7]:

1. Computer vision.

2. Prediction.

3. Semantic analysis.

4. Natural language processing. 
5. Information retrieval.

Of these five domains, computer vision, prediction, and information retrieval have applications in AM. More varied exploration into these areas has been enabled by recent advances in graphics hardware which have allowed for faster optimisation of ML algorithms on large training sets [7]. These advances have allowed for the implementation of ML solutions within AM environments.

Across design, production, and process, improvements to current practices in AM require significant expertise in operators and designers [8]. To leverage the benefits of AM, the design, process, and production become significantly more complex [8]. In design, mass customisation requires deep knowledge of the links between the variables being changed as well as the requirements of the part. Similarly, increasing part complexity, either to lighten the weight or deliver improved performance, greatly increases the difficulty in designing suitable part topologies. As a result, these goals often come with large time and/or computation trade-offs.

There is little orthogonalisation in AM parameters: for example, in material extrusion, increasing extrusion temperature may improve layer adhesion but may also increase stringing. As a result, optimising process parameters for specific parts or new materials can be a time consuming and costly procedure [9]. Furthermore, part consistency is essential in sectors where AM adoption is most likely, such as aerospace, but variation in part quality both between and within machines and builds presents a barrier to more widespread adoption. Variations can include inconsistent part geometries, porosity, and functional performance. These issues encompass the management and interpretation of large amounts of data and knowledge. Such problems may be eased through the proper application of ML methods by reducing the amount of human or computational effort required to deliver satisfactory results.

\section{Additive Manufacturing}

This section details the various AM processes and use cases to provide an understanding of the current position of AM and the barriers limiting adoption. This understanding will be further discussed in Section 4. There are a number of different AM processes currently available-ASTM categorises these into seven types [1]:

1. Binder jetting: a liquid bonding agent or adhesive is selectively deposited to join powdered materials together.

2. Directed energy deposition (DED): focused thermal energy (e.g., laser, electron beam, plasma arc) is used to fuse materials by melting as they are deposited.

3. Material extrusion: material is selectively dispensed through a nozzle or orifice onto a substrate.

4. Material jetting: droplets of build material are selectively deposited.

5. Powder bed fusion (PBF): thermal energy selectively fuses regions of a powder bed.

6. Sheet lamination: sheets of material are bonded layer-upon-layer to form a part.

7. Vat photopolymerisation: a vat of liquid photopolymer is selectively cured by lightactivated polymerisation.

There are three primary reasons for the adoption of AM techniques, including production of more complex geometries (than are achievable with conventional manufacturing approaches) without increasing cost, mass customisation of components, and supply chain disintermediation.

\subsection{Complex Geometries}

AM's layer-by-layer approach to manufacturing means that it can produce geometries that would be impossible for traditionally recognised manufacturing techniques. This has three main applications: light-weighting, performance optimisation and part consolidation.

Light-weighting may be achieved through topology optimisation (TO) or latticisation. In TO, a part is analysed based on an objective function, such as stiffness, and material is removed from the design that contributes least towards this function. This produces 
a more topologically complex part that can more precisely, and with less mass, meet its specification. Latticisation involves permeating a unit cell throughout the internal volume of a part. This can be done to remove mass but may also be leveraged to produce custom material properties or improve biofunctionality, such as with the design of auxetic structures for implants [10].

Fundamentally altering a part with a design for the additive manufacturing (DfAM) approach can enable further performance optimisation that would be impossible if AM was not used as the manufacturing technology of choice. Using hydraulic manifolds as an example, traditional designs may use drilled out through holes and plugs to create the required internal channels. This creates undesirable energy and pressure losses whilst resulting in parts with unnecessarily large masses [11]. By redesigning the manifolds for AM, without the constraints of straight, orthogonally intersecting channels, pressure losses have been found to reduce by up to $29.6 \%$ [11] and part mass has been reduced by as much as $91 \%$ [12]. Despite this, recognising which features require redesign as well as how to go about said redesign is very often dependent on the expertise of the designer.

Many large assemblies are designed in that way due to limitations in the ability of the manufacturing process to produce complex geometries (e.g., tool paths and undercuts). Reducing the number of parts in an assembly can reduce maintenance requirements, lead-time, weight, and production and non-recurring costs. A case study for AM partconsolidation is presented in Table 1. While the benefits of part consolidation are tangible, it may be difficult for designers, especially those inexperienced in AM, to select appropriate candidate parts.

Table 1. Profitability analysis of the effects of AM-enabled part consolidation of a high-bandwidth, direction tracking antenna array carried out by Optisys LLC [13].

\begin{tabular}{cc}
\hline Item & Improvement \\
\hline Part count & 100 pieces down to a 1-piece integrated assembly \\
Lead time & 11 months down to 2 months \\
Weight & $95 \%$ savings \\
Production costs & $20-25 \%$ reduction \\
Non-recurring costs & $75 \%$ reduction \\
\hline
\end{tabular}

\subsection{Mass Customisation}

$\mathrm{AM}^{\prime}$ s lack of reliance on tooling combined with its expensive materials and low production speeds (relative to feedstocks for more conventional manufacturing processes) make it best suited for high-value parts with low production volumes. This feature of AM is most beneficial when the part under consideration is bespoke, thus enabling cost effective production of parts customised for an individual consumer's needs or wants. The three sectors where this is most commonly used are medical/dental, packaging, and consumer products [3]. A selection of examples from each industry is summarised in Table 2.

Table 2. Prominent case studies of mass customization across its three primary application areas.

\begin{tabular}{ccc}
\hline Sector & Case Study & Source(s) \\
\hline Medical & Bespoke hearing aids & {$[14]$} \\
& $\begin{array}{c}\text { Hard and soft tissue biomedical implants } \\
\text { Custom casts, splints, orthotics, and prostheses }\end{array}$ & {$[3]$} \\
& Custom-fit 'direct digital packaging' & {$[3]$} \\
\hline Packaging & Custom-fit running shoes and insoles & {$[16,17]$} \\
\hline Consumer products &
\end{tabular}

While scanning allows for conformal shapes to be easily designed into a part [14], sophisticated design of the part's mesostructure is dependent on designer experience and computationally expensive tools, providing a hurdle for mass customised parts due to the lack of tools for this task. 


\subsection{Supply Chain Disintermediation}

Due to its ability to manufacture parts on-site, AM allows for lean and agile manufacturing. This is particularly of note for requirements such as spare parts, where the demand is highly variable. Liu et al. [18] concluded that AM has the ability to increase supply chain efficiency for spare parts in aerospace [18] while Hernandez et al. [19] concluded that AM has the ability to significantly reduce supply chain disruption for the United States Navy. These benefits may be further leveraged should the production of AM parts be made more repeatable through greater part consistency and geometrical accuracy.

\section{Machine Learning}

This section details the different types of ML and outlines common use cases relevant to AM to highlight the potential of the field when applied to AM limitations.

Supervised learning algorithms fit hypotheses to labelled training datasets, those where there is a known output. The trained algorithm may then be applied to unlabelled cases to predict the corresponding label. Supervised learning is itself split into two categories: classification and regression. Classification problems have qualitative labels, i.e., classes such as whether an image is that of a cat or not, whereas regression problems have quantitative labels, such as estimating the age of a cat based on an image [20].

Neural networks (NNs) are popular tools for supervised learning, especially with large datasets. These algorithms seek to emulate a brain by implementing layers of connected neurons, as shown in Figure 1. NNs map an input space onto an output space, which is usually of different dimensions. The implementation of NNs allows for non-linear decision boundaries to be inferred in a computationally efficient manner. More specialised NNs also exist for specific application areas. For example, convolutional neural networks (CNNs) utilise convolutional layers to identify features that may be present throughout the input space. CNNs are most often used in computer vision tasks, where similar features, for example vertical lines, may occur anywhere in the input space.

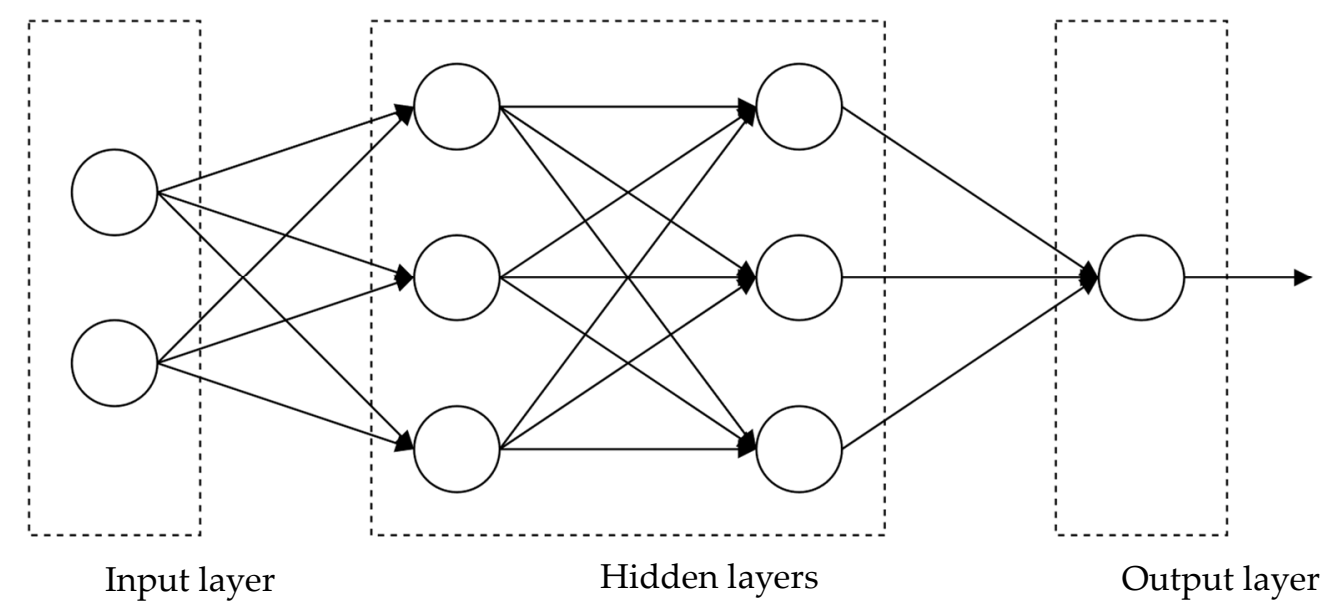

Figure 1. A fully connected NN with one input layer, two hidden layers, and one output layer. Circles represent neurons, which apply activation functions to the total sum of the products of the previous layer's activation and weight pairs.

Support vector machines (SVMs) are used in supervised learning tasks: traditionally classification but they are capable of regression. They calculate relationships between data in a higher dimensional space using a kernel function and placing a hyperplane decision boundary between classes to maximise the margins [20]. In regression tasks, the hyperplane is selected to best fit the data [20]. SVMs perform well for high-dimensional data but when there are far more features than training examples, they are prone to overfitting the training data. However, this can often be overcome through careful selection of an appropriate kernel function or through regularisation. 
Unsupervised learning problems attempt to infer patterns from unlabelled data. Since these problems do not have a label to compare against, they are often more difficult to evaluate. There are various types of unsupervised learning algorithms, with the two most common types being clustering and association rules. Clustering, also called data segmentation, algorithms group data into clusters. The data within each cluster are such that they are more closely related to each other than any data in a different cluster [20]. Association rule analysis, often called market basket analysis, seeks to identify prototype values for a feature set such that the probability density at those values is relatively large [20].

Reinforcement learning considers the optimisation of an agent's interaction with its environment through a reward signal [6]. While supervised learning extrapolates its output from a set of known scenarios, reinforcement learning operates without known training scenarios for the reward signal [6]. Despite this, reinforcement learning is disparate from unsupervised learning in that it is not attempting to discern hidden structure within the data but optimises said reward signal [6].

\section{Machine Learning for Additive Manufacturing}

This section presents prominent research and findings of ML applications in AM. Applications have been grouped by their area of application with AM, in accordance with Wang et al. [8].

\subsection{Machine Learning for Design for Additive Manufacturing}

Multiple studies, notably by Sosnovik and Oseledets [21], have utilised ML tools to accelerate the TO process. This was enabled through the implementation of CNNs whereby:

1. The input volume was the result of the topology optimisation after just a few iterations of the Solid Isotropic Material with Penalisation (SIMP) algorithm.

2. The output volume was a prediction of the part topology after 100 iterations of the SIMP algorithm.

Whilst not removing the need for the SIMP algorithm, the method presented by Sosnovik and Oseledets [21], demonstrated that ML can be effective in drastically reducing the computational workload required to run TO. Results ranged from $92 \%$ intersection over union accuracy for input volumes of just five SIMP iterations, to $99.2 \%$ after 80 [21]. This work has been extended in further studies by Banga et al. [22] and later by Harish et al. [23], which looked at 3D implementations for cantilevered beams, with Banga et al. [22] obtaining a binary accuracy of $96 \%$. The implications of these studies show that effective implementation of ML into the TO workflow may allow for:

1. A reduction in the time taken to carry out TO with a similar quality of output.

2. Higher resolution TO being efficiently used in practice thus enabling superior quality outputs.

3. Greater complexity in constraints applied, such as those specific to AM (e.g., supportless structures).

Biomimetic designs are enabled by AM but it is a major challenge to generate metamaterial structures with desired properties. Gu et al. effectively utilised CNNs to be able to generate structures with predictable strength and toughness [24]. This study built up three unit-cell "building blocks" from two jetted materials: one hard and one soft. These three building blocks were then randomly assigned to each of the 64 cells in an $8 \times 8$ array and then simulated to create a training example. In total, 80,000 training examples, or $10^{-8 \%}$ of the possible combinations, were created to train the $\mathrm{CNN}$ which was able to predict material properties. The model had a normalised root mean square deviation from the simulated results of 0.4926 on the test set. AM specimens were then produced to validate these findings. Here, the use of ML allowed the researcher to accurately screen the whole design space in hours compared to years for conventional simulation-only approaches [24]. 
Recent works have also leveraged NNs to generate lattice structures based on required mechanical properties. Jiang et al. developed a methodology for designing an ML implementation to determine optimal design geometries to produce desired properties, for example, stress-strain curves [25]. The methodology was implemented through the design of an ankle brace with three zones, each with its own distinct mechanical requirements: maximum torque, range of motion, as well as stress and strain in two orientations. An $\mathrm{NN}$ was developed to define the design parameters for a pre-determined unit cell that would meet these requirements. To train said network, simulations of random sets of the design parameters within acceptable ranges were carried out to determine their stressstrain response under the given maximum torque. This was then used as training data for their model. The resulting regression model, with the same amount of data available, was capable of generating structures with comparable errors to conventional methods, but with improved computational efficiency [25]. Due to the nature of the NN architecture used, however, performance could likely be further improved through gathering additional data.

An additional problem faced in DfAM is in knowledge dissemination: helping designers learn how to best design their parts for fabrication using AM technologies [3]. With the number of AM-specific design features growing, tools for designers are increasingly required [26]: ML has successfully been implemented in creating such tools. Yao et al. developed a flexible hybrid ML tool to recommend design features to inexperienced designers based on three designer-coded categories: loadings, objectives, and properties [26]. The tool produced a dendrogram for the designer's part and compared it against a hierarchically clustered database of existing design features [26]. An SVM was then used to target features based on their similarity to the designer's coding for the part [26]. Despite only considering functionality-specific features, after testing it with inexperienced designers, the tool was found to effectively enable the exploration of AM design freedoms [26].

\subsection{Machine Learning for Additive Manufacturing Process}

Within the domain of AM processes, ML is applied in two main areas: parameter optimisation and process monitoring [8]. This section will outline the current states of these areas.

\subsubsection{Parameter Optimisation}

Process parameter optimisation is often a manual and time-consuming process, making it costly. Similarly, manual process monitoring/control also creates additional costs. Since manual parameter optimisation requires the production of large numbers of samples, there is readily available data for the production of ML tools. Said tools, which make up a plurality of the research on ML for AM [27], largely take the route of optimising key parameters for a particular quality indicator or set of indicators [8]. PBF and material extrusion are the most common AM technologies to have had this type of tool developed.

Many studies have been carried out to optimise parameters for powder bed fusion processes. A summary of the parameters studied as well as respective quality indicators are shown in Figure 2, with porosity and fatigue life identified as having the largest variety of process parameters linked to them. In addition, porosity is the leading quality indicator in the research, with at least five studies implementing machine learning algorithms to optimise process parameters [28-32]. Liu et al. [32] built on previous efforts to develop a "physics-informed" model rather than a conventional "setting" model. The two model types, along with a third combined model, were found to provide similar performance to one another (within error margin). In addition, the novel "physics-informed" model was identified as being more easily generalised to other machines, although this was not tested. 


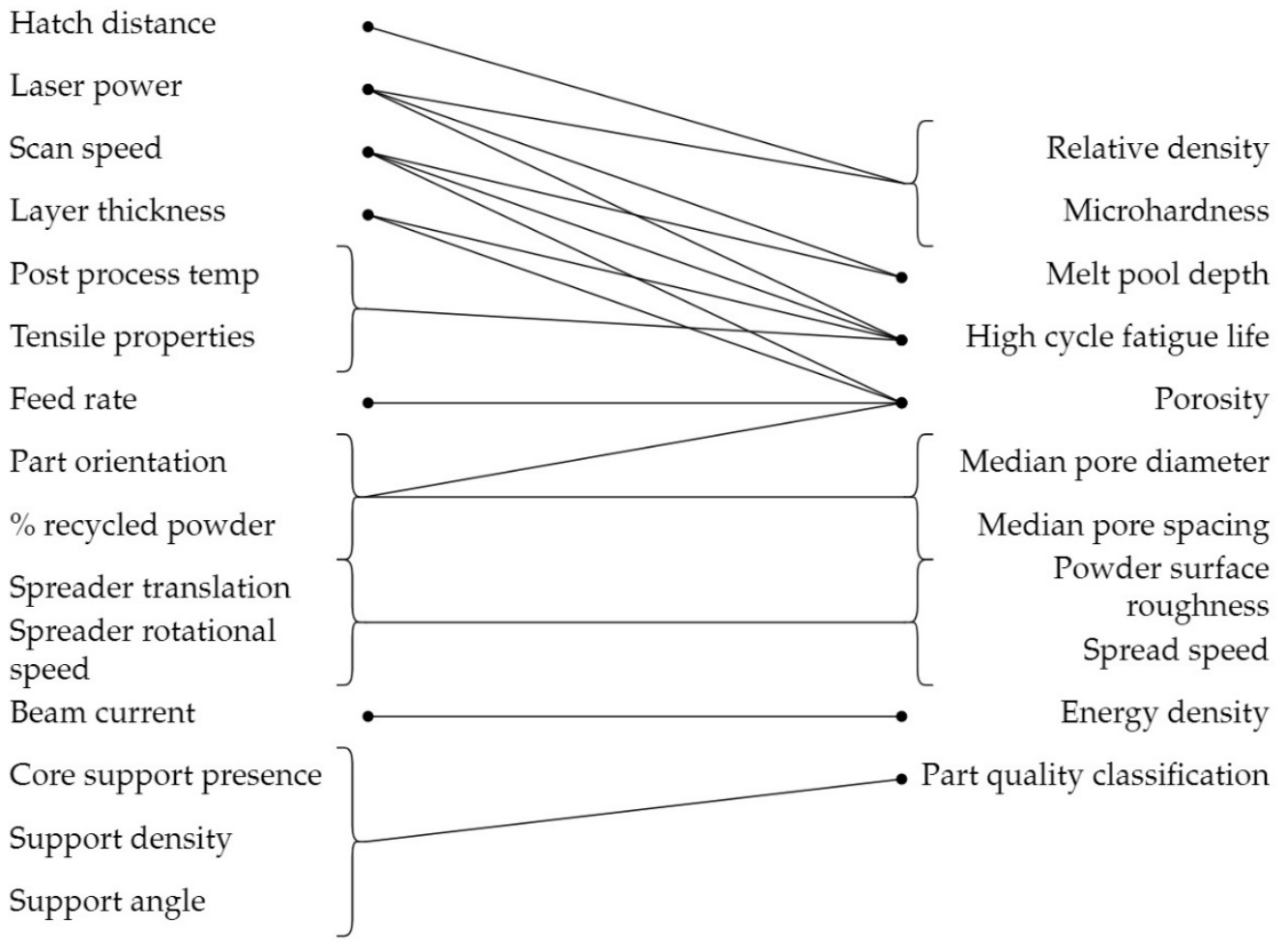

Figure 2. Matching diagram relating PBF process parameters to quality indicators from successful implementations. Adapted from Wang et al. [8].

Many studies have also been carried out to predict and/or optimise quality indicators for the material extrusion process. Figure 3 shows a summary of which process parameters have been linked to which quality indicators. Comparing Figure 2 to Figure 3, it can be seen that a much greater number of parameters are considered for each quality indicator in material extrusion over PBF. This may be attributed to lower part costs in material extrusion over $\mathrm{PBF}$, thus allowing for more training samples and potentially more complex ML implementations. NNs can capture non-linearities more easily than simpler regression models, making them the prevailing algorithm for material extrusion process parameter studies.

A key area for optimisation in material extrusion is component surface roughness [4]. Li et al. [33] built a predictive model for surface roughness based on build plate and extruder temperature, build plate and extruder vibration, and melt pool temperature. The model used AdaBoost, a learning algorithm that is an ensemble of various weaker algorithms, and was able to predict surface roughness with a root mean square error of $0.7 \mu \mathrm{m}[33]$.

DED processes have also had parameters optimised as discussed in the literature. The bulk of these works focus on controlling aspects of the melt pool and resulting tracks, including: track width and height [34-36], melt pool geometry [37], and thermal history of the melt pool [38]. More recent works have studied the properties of the final part: Narayana et al. [39] built a NN to predict built part height and density from laser power, scan speed, powder feed rate, and layer thickness. It was found that these parameters were all of significant importance for density whereas scan speed and feed rate had the largest effect on build height. These findings were reinforced by the model's prediction accuracy of $99 \%$. Similar to material extrusion, DED produces parts with poor surface roughness [40]. Xia et al. [40], used an NN to model and predict surface roughness based on overlap ratio, welding speed, and wire feed speed with a root mean square error of $6.94 \%$. A small training set was identified as a major limiter on the model's accuracy [40]. 
\# contours

Layer thickness

Air gap

Raster angle

Build orientation

Road width

Print temperature

Print speed

Cooling fan speed

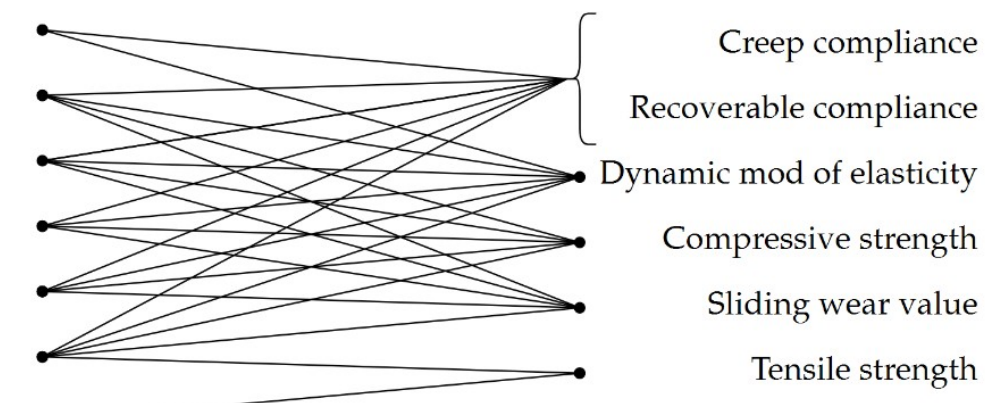

Figure 3. Matching diagram relating material extrusion process parameters to quality indicators from successful implementations. Adapted from Wang et al. [8].

ML for process parameter optimisation in binder jetting has a lesser volume of literature associated with these aspects: Chen and Zhao [41] developed an NN powered software tool to recommend layer thickness, printing saturation, heater power ratio and drying time based on user defined preference for surface roughness as well as dimensional accuracy along the $Y$ and $Z$ axes. Their software also predicted surface roughness as well as $\mathrm{Y}$ and $\mathrm{Z}$ shrinkage. The error in these predictions was found to be $1.98 \%, 5.38 \%$, and $16.58 \%$, respectively. It was suggested that improvements could be made through use of a larger training set or by reducing the model's independence by implementing an element of physical modelling [41].

\subsubsection{Process Monitoring}

While parameter optimization may help to improve process predictability, it cannot eliminate failures entirely [42]. With print failures contributing significantly to the cost of AM parts [43], process monitoring techniques able to detect build failures and defects are necessary. Various ML implementations have sought to solve this problem and fall into two categories depending on their input data type: optical and acoustic [8].

Optical monitoring solutions are the most widely used, with the data often coming from digital, high speed, or infrared cameras [8]. In PBF processes, where the bulk of monitoring research is currently concentrated, the most common target of these computer vision tasks is the melt pool. From thermal data of the melt pool, Kwon et al. [42] trained a CNN-based program to differentiate between high, medium, and low quality builds with a failure rate of under $1.1 \%$ [42], allowing for potential time and cost savings. Other works have used optical data from laser melting plumes [44-46] for similar quality classification tasks, with Zhang et al. $[45,46]$ finding that the best results are achieved when melt pool, plume, and spatter data are used together to classify part quality. The most recent work found a type of NN called a long-short term memory network, to be most effective in prediction, with a root mean squared error of $13.9 \%$ [46].

Optical monitoring has also been implemented for other AM processes, including binder jetting and material extrusion. Gunther et al. [47] used optical monitoring to detect faults in binder jet parts based on the frequency and density of brightly coloured pixels. The algorithms employed here were not provided, nor was there discussion of the accuracy of the employed model. In material extrusion, optical monitoring has been implemented for in situ defect detection [48]. Wu et al. [48] used a classification algorithm to identify the presence of infill print defects in material extrusion, allowing for greater confidence in final part's quality. The study achieved an accuracy of over $95 \%$ but did not consider other vital quality indicators such as precision and recall. Li et al. [49] used in situ optical monitoring 
of a material extrusion process to determine dimensional deviation with zero mean error and a standard deviation of $0.02 \mathrm{~mm}$.

Acoustic monitoring is a newer and less widely adopted method of monitoring a build mid-print. These techniques rely on characteristic acoustic signals that relate to part porosity [50] and melt states [51] in PBF processes as well as process failures in material extrusion [52]. Advantages of acoustic monitoring solutions include the lower cost sensors compared to optical monitoring techniques [50]. ML algorithms applied also vary from supervised CNNs to clustering solutions. Researchers in these fields have achieved confidence of up to $89 \%$ for porosity classification [50] and $94 \%$ for melt-pool related defects [51], showing acoustic monitoring to be an effective method for flagging problem builds with less need for post-print examination and testing [51]. Acoustic monitoring has also been applied to material extrusion processes. Wu et al. [53] used acoustic monitoring and an SVM classifier to determine if the extruder was successfully pushing out material with an accuracy of $100 \%$. The SVM was also able to identify extruder blockages (normal, semi-blocked, or blocked) with a $92 \%$ accuracy.

\subsection{Machine Learning for Additive Manufacturing Production}

In AM production, as well as implementing strategies discussed in Sections 4.1 and 4.2, additional tools have been developed to aid in general production planning using NNs, and manufacturability through a variety of methods. In addition, research has been conducted on ML implementations to replicate CAD geometry from acoustic signals produced during manufacturing, creating concerns around data security [54,55].

\section{Printability and Dimensional Deviation Management}

Models have been developed to aid in the identification of printability of components in material extrusion [56] and PBF processes [57] utilising CNNs and SVMs, respectively. The use of NNs has also been shown to aid in reducing print time estimation error for PBF processes from $20-35 \%$ to $2-15 \%$ [58], enabling improved management of these machines.

There are three sources of dimensional deviation in AM parts [59]: the material (shrinkage and warpage), the machine, and the file preparation (e.g., resolution reduction due to conversion from CAD model to STL file format). ML has been used to correct these in PBF [60], where NNs were implemented to first optimise part orientation, reducing deviation due to the machine, then modify CAD geometry to account for thermal effects on the material.

Khanzedah et al. [61] implemented an unsupervised learning algorithm, a self-organising map, to analyse and assess point-cloud data for the dimensional deviation of parts made with material extrusion processes. Their implementation was able to sort the part's deviations into discrete clusters based on the severity of the deviations present, which allowed sub-optimal process conditions to be identified. Noriega et al. [62] used NNs to compensate for dimensional deviation by modifying the part's scale for material extrusion. Their two NN implementations were used to reduce deviation by $50 \%$ for external dimensions and $30 \%$ for internal dimensions. In a more robust study using the AM of a T4 spinal vertebrae as a case study, Charalampous et al. [63] were able to achieve a $25 \%$ reduction in dimensional deviation at a $1: 1$ scale and $33 \%$ at a $3: 1$ scale.

Other studies have looked to correct dimensional deviation in binder jetting and DED processes. Shen et al. [64] presented a study using CNNs to predict dimensional deviation and compensate for it via translation, scaling, and rotation of dental crown CAD geometry to be manufactured by binder jetting processes. The $\mathrm{CNN}$ used a voxel-based approach where, in the analysis of the implementation, each voxel was deemed to be correct or incorrect. This was then used to generate F1 scores: a single-value metric used to evaluate the model's recall and precision. The predictive model and compensatory model both averaged $94 \%$ for their F1 scores. Despite this, no physical samples were manufactured so these findings are unverified. 
In DED, the dominant method of dimensional deviation correction is to optimise the geometry of individual tracks through the optimisation of process parameters $[33,35,36,64]$. This partially corrects material and machine errors but not those associated with the file preparation process. Caiazzo and Caggiano [37] developed one such model to tune laser power, scanning speed, and powder feed rate to achieve a specified track geometry with mean absolute errors of $2.0 \%, 5.8 \%$, and $5.5 \%$, respectively. An alternative approach was presented by Choi who built a predictive $\mathrm{NN}$ for multi-track height based on laser power, powder feed rate, and coaxial gas flow rate with an accuracy of $96.63 \%$. Choi [65] queried their model for optimal results: $300 \mathrm{~W}, 3.7 \mathrm{~g} / \mathrm{min}$, and $61 / \mathrm{min}$, respectively.

The vulnerability of material extrusion machines to intellectual property theft was identified by Al Faruque et al. [54] and Hojjati et al. [55]. Al Faruque et al. [54] showed that the noise emitted by stepper motors during printing can be recorded and processed to infer features of the print process:

1. Axis of motion.

2. Direction of motion.

3. Speed of hot-end motion

4. Distance travelled in each axis.

5. Extrusion amount.

This information was used in a physical-to-cyber attack to reconstruct the CAD geometry being fabricated. Both studies utilised non-descript supervised classification and regression models to determine the features and feature values, respectively. Geometry was successfully reconstructed with an average axis prediction accuracy of $78 \%$ and an average length prediction error of $18 \%$ [54]. Unlike most applications discussed, IP theft is an aspect of AM that is harmed by ML. This must be resolved if AM, particularly material extrusion, is to be made viable for security-sensitive applications.

\section{Conclusions and Prospects}

In research, ML has been shown to be effective in furthering AM design, process, and production. In AM design, ML has been used to accelerate tools, explore new materials, enable the identification of property-structure relationships, and aid novice designers. TO acceleration and material exploration have limited scope in their current states and need further development to work with larger design spaces or with finer spatial resolutions. Property-structure relationships have the potential to be useful in functional lattice design, but current implementations have inadequate transitional regions and accuracies that may be too low for some industries. There are also insufficient case studies to support further adoption of these techniques. Design feature recommenders in their current forms are quite robust but require curation to remain relevant and, like functional lattice design systems, need further case studies to be developed to support their adoption. In AM process, most work focuses on process parameter optimisation. These are effective in optimising process parameters for one or multiple quality indicators. Despite this, these optimisers are machine specific, and no studies have been identified that attempt to produce more general models. Without such developments, many samples will have to be produced whether the final process used for optimisation is manual or through ML. ML has been successfully used to improve the predictability of AM production techniques but has also been shown to be able to exploit AM processes and enable IP theft. While there have been no real-world reports of this sort of theft taking place, it remains a hurdle to be solved to make AM, particularly material extrusion, a secure process.

Overall, ML has had a positive impact on the prospects of furthering AM adoption and improving its value proposition. That said, most ML applications for AM are not robust or trusted enough to be adopted in industry. As a result, research efforts should focus on further developing these tools for real-world use and reporting industry case studies to build confidence in their efficacy. 
Author Contributions: Conceptualization, D.G. and A.E.W.R.; formal analysis, D.G.; investigation, D.G.; resources, D.G.; writing-original draft preparation, D.G.; writing-review and editing, A.E.W.R. and S.D.Q.; visualization, D.G.; supervision, A.E.W.R. and S.D.Q.; project administration, D.G. and A.E.W.R. All authors have read and agreed to the published version of the manuscript.

Funding: This research was funded by the European Regional Development Fund, through the Greater Innovation for Smart Materials Optimisation (GISMO) Project (grant reference number 03R18P02671).

Data Availability Statement: Dara sharing not applicable.

Conflicts of Interest: The authors declare no conflict of interest.

Entry Link on the Encyclopedia Platform: https:/ / encyclopedia.pub/13191.

\section{References}

1. ISO/ASTM. ISO/ASTM52900-15, Standard Terminology for Additive Manufacturing-General Principles—Terminology. ASTM Int. 2015, 1, 1-9. [CrossRef]

2. Ahlers, D.; Wasserfall, F.; Hendrich, N.; Zhang, J. 3D printing of nonplanar layers for smooth surface generation. In Proceedings of the IEEE International Conference on Automation Science and Engineering, Vancouver, BC, Canada, 22-26 August 2019; pp. 1737-1743. [CrossRef]

3. Thompson, M.K.; Moroni, G.; Vaneker, T.; Fadel, G.; Campbell, R.I.; Gibson, I.; Bernard, A.; Schulz, J.; Graf, P.; Ahuja, B.; et al. Design for Additive Manufacturing: Trends, opportunities, considerations, and constraints. CIRP Ann. Manuf. Technol. 2016, 65, 737-760. [CrossRef]

4. Gibson, I.; Rosen, D.; Stucker, B.; Khorasani, M. Additive Manufacturing Technologies, 3rd ed.; Springer: Berlin/Heidelberg, Germany, 2021; ISBN 978-3-030-56127-7. [CrossRef]

5. Dowling, L.; Kennedy, J.; O'Shaughnessy, S.; Trimble, D. A review of critical repeatability and reproducibility issues in powder bed fusion. Mater. Des. 2020, 186, 108346. [CrossRef]

6. Sutton, R.S.; Barto, A.G. Reinforcement Learning: An Introduction, 2nd ed.; The MIT Press: Cambridge, MA, USA, 2018; ISBN 9780262039246.

7. Shinde, P.P.; Shah, S. A Review of Machine Learning and Deep Learning Applications. In Proceedings of the 2018 4th International Conference on Computing, Communication Control and Automation (ICCUBEA), Pune, India, 16-18 August 2018. [CrossRef]

8. Wang, C.; Tan, X.P.; Tor, S.B.; Lim, C.S. Machine learning in additive manufacturing: State-of-the-art and perspectives. Addit. Manuf. 2020, 36, 101538. [CrossRef]

9. Qi, X.; Chen, G.; Li, Y.; Cheng, X.; Li, C. Applying Neural-Network-Based Machine Learning to Additive Manufacturing: Current Applications, Challenges, and Future Perspectives. Engineering 2019, 5, 721-729 101016/jeng201904012. [CrossRef]

10. Darwish, S.M.H.; Aslam, M.U. Auxetic cellular structures for custom made orthopedic implants using additive manufacturing. Int. J. Eng. Adv. Technol. 2014, 4, 2249-8958.

11. Xie, G.; Dong, Y.; Zhou, J.; Sheng, Z. Topology optimization design of hydraulic valve blocks for additive manufacturing. Proc. Inst. Mech. Eng. Part C J. Mech. Eng. Sci. 2020, 234, 1899-1912. [CrossRef]

12. Diegel, O.; Schutte, J.; Ferreira, A.; Chan, Y.L. Design for additive manufacturing process for a lightweight hydraulic manifold. Addit. Manuf. 2020, 36, 101446. [CrossRef]

13. GE Additive. For the Ready. Launch Your Additive Manufacturing Program with Help from GE Additive. 2020. Available online: https:/ / www.ge.com/additive/sites/default/files/2020-11/GE_Space_Brochure_8.5x11_R5_0.pdf (accessed on 16 June 2021).

14. Redwood, B.; Schoffer, F.; Garret, B. The 3D Printing Handbook: Technologies, Design and Applications, 1st ed.; 3D Hubs: Amsterdam, The Netherlands, 2018; ISBN 9789082748505.

15. Chen, R.K.; Jin, Y.-A.; Wensman, J.; Shih, A. Additive manufacturing of custom orthoses and prostheses-A review. Addit. Manuf. 2016, 12, 77-89. [CrossRef]

16. EOS Shoe Soles from the 3D Printer/EOS GmbH. Available online: https://www.eos.info/en/3d-printing-examplesapplications / people-health/sports-lifestyle-consumer-goods/shoes-soles (accessed on 28 May 2021).

17. Dong, G.; Tessier, D.; Zhao, Y.F. Design of Shoe Soles Using Lattice Structures Fabricated by Additive Manufacturing. Proc. Des. Soc. Int. Conf. Eng. Des. 2019, 5-8. [CrossRef]

18. Liu, P.; Huang, S.H.; Mokasdar, A.; Zhou, H.; Hou, L. Production Planning \& Control The Management of Operations The impact of additive manufacturing in the aircraft spare parts supply chain: Supply chain operation reference (scor) model based analysis. Prod. Plan. Control. 2014, 25, 1169-1181. [CrossRef]

19. Hernandez, B.R.; Housel, T.; Ford, D. An Investigation into the Use of $£ D$ Scanning and PRinting Technologies in the Navy Collaborative Product Lifecycle Management. Master's Thesis, Naval Postgraduate SCHOOL, Monterey, CA, USA, 2013. Available online: https: / calhoun.nps.edu/bitstream/handle/10945/38945/13Dec_Hernandez_Benjamin.pdf?sequence=1\& isAllowed $=\mathrm{y}$ (accessed on 16 June 2021).

20. Hastie, T.; Tibshirani, R.; Friedman, J. Springer Series in Statistics The Elements of Statistical Learning Data Mining, Inference, and Prediction, 2nd ed.; Springer: Berlin/Heidelberg, Germany, 2008; ISBN 978-0-387-84857-0. [CrossRef] 
21. Sosnovik, I.; Oseledets, I. Neural networks for topology optimization. Russ. J. Numer. Anal. Math. Model. 2019, 34, $215-223$. [CrossRef]

22. Banga, S.; Gehani, H.; Bhilare, S.; Patel, J.; Kara, B. 3D Topology Optimzation Using Convolutional Neural Networks. arXiv 2018, arXiv:1808.07440.

23. Harish, B.; Eswara Sai Kumar, K.; Srinivasan, B. Topology optimization using convolutional neural network. In Proceedings of the Lecture Notes in Mechanical Engineering; Springer: Berlin/Heidelberg, Germany, 2020; pp. 301-307. [CrossRef]

24. Gu, G.X.; Chun-Teh Chen, A.; Richmond, D.J.; Buehler, M.J. Bioinspired hierarchical composite design using machine learning: Simulation, additive manufacturing, and experiment. Mater. Horizons 2018, 5, 939. [CrossRef]

25. Jiang, J.; Xiong, Y.; Zhang, Z.; Rosen, D.W. Machine learning integrated design for additive manufacturing. J. Intell. Manuf. 2020, 1-14. [CrossRef]

26. Yao, X.; Moon, S.K.; Bi, G. A hybrid machine learning approach for additive manufacturing design feature recommendation. Rapid Prototyp. J. 2017, 23. [CrossRef]

27. Mahmood, M.A.; Visan, A.I.; Ristoscu, C.; Mihailescu, I.N. Artificial Neural Network Algorithms for 3D Printing. Materials 2021, 14, 163. [CrossRef] [PubMed]

28. Silbernagel, C.; Aremu, A.; Ashcroft, I. Using machine learning to aid in the parameter optimisation process for metal-based additive manufacturing. Rapid Prototyp. J. 2019, 26, 625-637. [CrossRef]

29. Kappes, B.; Moorthy, S.; Drake, D.; Geerlings, H.; Stebner, A. Machine learning to optimize additive manufacturing parameters for laser powder bed fusion of Inconel 718. In Proceedings of the Minerals, Metals and Materials Series; Springer International Publishing: Berlin/Heidelberg, Germany, 2018; Volume 2018-June, pp. 595-627. [CrossRef]

30. Garg, A.; Lam, J.S.L. Measurement of environmental aspect of 3-D printing process using soft computing methods. Meas. J. Int. Meas. Confed. 2015, 75, 210-217. [CrossRef]

31. Garg, A.; Lam, J.S.L.; Savalani, M.M. A new computational intelligence approach in formulation of functional relationship of open porosity of the additive manufacturing process. Int. J. Adv. Manuf. Technol. 2015, 80, 555-565. [CrossRef]

32. Liu, R.; Liu, S.; Zhang, X. A physics-informed machine learning model for porosity analysis in laser powder bed fusion additive manufacturing. Int. J. Adv. Manuf. Technol. 2021, 113, 1943-1958. [CrossRef]

33. Li, Z.; Zhang, Z.; Shi, J.; Wu, D. Prediction of surface roughness in extrusion-based additive manufacturing with machine learning. Robot. Comput. Integr. Manuf. 2019, 57, 488-495. [CrossRef]

34. Xiong, J.; Zhang, G.; Hu, J.; Wu, L. Bead geometry prediction for robotic GMAW-based rapid manufacturing through a neural network and a second-order regression analysis. J. Intell. Manuf. 2014, 25, 157-163. [CrossRef]

35. Li, Y.; Sun, Y.; Han, Q.; Zhang, G.; Horváth, I. Enhanced beads overlapping model for wire and arc additive manufacturing of multi-layer multi-bead metallic parts. J. Mater. Process. Technol. 2018, 252, 838-848. [CrossRef]

36. Lu, Z.; Li, Ã.; Lu, B.; Zhang, A.; Zhu, G.; Pi, G. The prediction of the building precision in the Laser Engineered Net Shaping process using advanced networks ARTICLE IN PRESS. Opt. Lasers Eng. 2010, 48, 519-525. [CrossRef]

37. Caiazzo, F.; Caggiano, A. Laser Direct Metal Deposition of 2024 Al Alloy: Trace Geometry Prediction via Machine Learning. Materials 2018, 11, 444. [CrossRef]

38. Mozaffar, M.; Paul, A.; Al-Bahrani, R.; Wolff, S.; Choudhary, A.; Agrawal, A.; Ehmann, K.; Cao, J. Data-driven prediction of the high-dimensional thermal history in directed energy deposition processes via recurrent neural networks. Manuf. Lett. 2018, 18, 35-39. [CrossRef]

39. Narayana, P.L.; Kim, J.H.; Lee, J.; Choi, S.-W.; Lee, S.; Park, C.H.; Yeom, J.-T.; Reddy, N.G.S.; Hong, J.-K. Optimization of process parameters for direct energy deposited Ti-6Al-4V alloy using neural networks. Int. J. Adv. Manuf. Technol. 2021, 114, 3269-3283. [CrossRef]

40. Xia, C.; Pan, Z.; Polden, J.; Li, H.; Xu, Y.; Chen, S. Modelling and prediction of surface roughness in wire arc additive manufacturing using machine learning. J. Intell. Manuf. 2021, 1-16. [CrossRef]

41. Chen, H.; Zhao, Y.F. Learning Algorithm Based Modeling and Process Parameters Recommendation System for Binder Jetting Additive Manufacturing Process. Proc. ASME Des. Eng. Tech. Conf. 2016, 1. [CrossRef]

42. Kwon, O.; Kim, G.H.; Ham, M.J.; Kim, W.; Kim, G.-H.; Cho, J.-H.; Kim, N.I.; Kim, K. Kangil A deep neural network for classification of melt-pool images in metal additive manufacturing. J. Intell. Manuf. 2020, 31, 375-386. [CrossRef]

43. Baumers, M.; Dickens, P.; Tuck, C.; Hague, R. The cost of additive manufacturing: Machine productivity, economies of scale and technology-push. Technol. Forecast. Soc. Chang. 2016, 102, 193-201. [CrossRef]

44. Ye, D.; Hsi Fuh, J.Y.; Zhang, Y.; Hong, G.S.; Zhu, K. In situ monitoring of selective laser melting using plume and spatter signatures by deep belief networks. ISA Trans. 2018, 81, 96-104. [CrossRef] [PubMed]

45. Zhang, Y.; Hong, G.S.; Ye, D.; Zhu, K.; Fuh, J.Y.H. Extraction and evaluation of melt pool, plume and spatter information for powder-bed fusion AM process monitoring. Mater. Des. 2018, 156, 458-469. [CrossRef]

46. Zhang, Z.; Liu, Z.; Wu, D. Prediction of melt pool temperature in directed energy deposition using machine learning. Addit. Manuf. 2021, 37, 101692. [CrossRef]

47. Gunther, D.; Pirehgalin, M.F.; Weis, I.; Vogel-Heuser, B. Condition monitoring for the Binder Jetting AM-process with machine learning approaches. In Proceedings of the 2020 IEEE Conference on Industrial Cyberphysical Systems (ICPS), Tampere, Finland, 10-12 June 2020; pp. 417-420. [CrossRef] 
48. Wu, M.; Phoha, V.V.; Moon, Y.B.; Belman, A.K. Detecting malicious defects in $3 \mathrm{~d}$ printing process using machine learning and image classification, ASME 2016 International Mechanical Engineering Congress and Exposition. Am. Soc. Mech. Eng. Digit. Collect. 2016. [CrossRef]

49. Li, L.; McGuan, R.; Isaac, R.; Kavehpour, P.; Candler, R. Improving precision of material extrusion 3D printing by in-situ monitoring \& predicting 3D geometric deviation using conditional adversarial networks. Addit. Manuf. 2021, $38,101695$. [CrossRef]

50. Shevchik, S.A.; Kenel, C.; Leinenbach, C.; Wasmer, K. Acoustic emission for in situ quality monitoring in additive manufacturing using spectral convolutional neural networks. Addit. Manuf. 2018, 21, 598-604. [CrossRef]

51. Ye, D.; Hong, G.S.; Zhang, Y.; Zhu, K.; Fuh, J.Y.H. Defect detection in selective laser melting technology by acoustic signals with deep belief networks. Int. J. Adv. Manuf. Technol. 2018, 96, 2791-2801. [CrossRef]

52. Wu, H.; Yu, Z.; Wang, Y. A New Approach for Online Monitoring of Additive Manufacturing Based on Acoustic Emission. ASME Int. 2016. [CrossRef]

53. Wu, H.; Wang, Y.; Yu, Z. In situ monitoring of FDM machine condition via acoustic emission. Int. J. Adv. Manuf. Technol. 2016, 84, 1483-1495. [CrossRef]

54. Abdullah Al Faruqye, M.; Chhetri, S.R.; Canedo, A.; Wan, J. Acoustic side-channel attacks on additive manufacturing systems. In Proceedings of the 7th International Conference on Cyber-Physical Systems (ICCPS '16), Vienna, Austria, 11-14 April 2016; pp. 1-10.

55. Hojjati, A.; Adhikari, A.; Struckmann, K.; Chou, E.J.; Nguyen, T.N.T.; Madan, K.; Winslett, M.S.; Gunter, C.A.; King, W.P. Leave your phone at the door: Side channels that reveal factory floor secrets. Proc. ACM Conf. Comput. Commun. Secur. 2016, 883-894. [CrossRef]

56. Tang, Y.; Dong, G.; Zhou, Q.; Zhao, Y.F. Lattice Structure Design and Optimization with Additive Manufacturing Constraints. IEEE Trans. Autom. Sci. Eng. 2018, 15, 1546-1562. [CrossRef]

57. Zhang, Y.; Dong, G.; Yang, S.; Zhao, Y.F. Machine learning assisted prediction of the manufacturability of laser-based powder bed fusion process. In Proceedings of the ASME Design Engineering Technical Conference; American Society of Mechanical Engineers (ASME), Anaheim, CA, USA, 18-21 August 2019; Volume 1. [CrossRef]

58. Munguía, J.; Ciurana, J.; Riba, C. Neural-network-based model for build-time estimation in selective laser sintering. Proc. Inst. Mech. Eng. Part B J. Eng. Manuf. 2009, 223, 995-1003. [CrossRef]

59. Omairi, A.; Ismail, Z.H. Towards machine learning for error compensation in additive manufacturing. Appl. Sci. 2021, 11, 2375. [CrossRef]

60. Chowdhury, S.; Mhapsekar, K.; Anand, S. Part Build Orientation Optimization and Neural Network-Based Geometry Compensation for Additive Manufacturing Process. J. Manuf. Sci. Eng. Trans. ASME 2018, 140. [CrossRef]

61. Khanzadeh, M.; Rao, P.; Jafari-Marandi, R.; Smith, B.K.; Tschopp, M.A.; Bian, L. Quantifying Geometric Accuracy with Unsupervised Machine Learning: Using Self-Organizing Map on Fused Filament Fabrication Additive Manufacturing Parts. J. Manuf. Sci. Eng. Trans. ASME 2018, 140. [CrossRef]

62. Noriega, A.; Blanco, D.; Alvarez, B.J.; Garcia, A. Dimensional accuracy improvement of FDM square cross-section parts using artificial neural networks and an optimization algorithm. Int. J. Adv. Manuf. Technol. 2013, 69, 2301-2313. [CrossRef]

63. Charalampous, P.; Kostavelis, I.; Kontodina, T.; Tzovaras, D. Learning-based error modeling in FDM 3D printing process. Rapid Prototyp. J. 2021, 27, 507-517. [CrossRef]

64. Shen, Z.; Shang, X.; Zhao, M.; Dong, X.; Xiong, G.; Wang, F.Y. A learning-based framework for error compensation in 3D printing. IEEE Trans. Cybern. 2019, 49, 4042-4050. [CrossRef]

65. Choi, T.-Y. Machine Learning Based Predictive Modelling of Dimensional Quality in Depostion with SUS316L; Graduate School of UNIST: Ulsan, Korea, 2020. 